\title{
The Effect of Preoperative Ultrasound-Guided Erector Spinae Plane Block on Chronic Postsurgical Pain After Breast Cancer Surgery: A Propensity Score-Matched Cohort Study
}

\author{
Ling Xin $\cdot$ Ning Hou $\cdot$ Ziyan Zhang $\cdot$ Yi Feng (D)
}

Received: October 3, 2021 / Accepted: November 5, 2021 / Published online: November 26, 2021

(c) The Author(s) 2021

\begin{abstract}
Introduction: The high incidence of chronic postsurgical pain (CPSP) has been a major issue after breast cancer surgery (BCS). The impact of regional anesthesia (RA) techniques on CPSP remains conflicting. In this propensity scorematched cohort study, we aimed to investigate the effect of preoperative single-shot erector spinae plane block (ESPB) adding to general anesthesia (GA) on the incidence of CPSP at 1 year following BCS.

Methods: Data of adult female patients who underwent unilateral BCS between October 2019 and June 2020 were retrospectively collected. Patients were grouped to ESPB combined with GA (ESPB + GA) and GA alone, respectively. All patients were prospectively followed up at 1 year after surgery. CPSP and neuropathic pain (NP) were measured using the brief pain inventory-short form (BPI-SF) and ID Pain scale. Eleven confounding factors were managed by propensity score matching (PSM) to achieve between-group balance. The primary outcome was the incidence of CPSP at 1 year after BCS. The secondary outcomes include proportion of $\mathrm{NP}$, severity, and interference of CPSP at 1 year
\end{abstract}

L. Xin $\cdot$ N. Hou $\cdot$ Z. Zhang $\cdot$ Y. Feng $(\bowtie)$ Department of Anesthesiology, Peking University People's Hospital, No. 11 Xizhimen South Street, Xicheng District, Beijing, China e-mail: doctor_yifeng@sina.com after surgery, acute postoperative pain, postoperative nausea and vomiting (PONV), hospital length of stay (LOS), and adverse events.

Results: After PSM, data for 194 patients were available for analysis (97 in each group). No significant difference in the incidence of CPSP $(P=1.000)$ nor percentage of patients with NP $(P=0.442)$ was found between the two groups. Both matched groups had similar intensity of CPSP $(P=0.547)$ measured by BPI-SF as well as the rates of moderate to severe CPSP $(P=1.000)$. A significant decrease in acute pain scores $(P=0.043)$ and rates of rescue analgesics demand $(P=0.042)$ were observed in the $\mathrm{ESPB}+\mathrm{GA}$ group compared to the GA group. Multivariate logistic regression on the total study cohort showed that axillary lymph node dissection (ALND) (OR 3.541, 95\% CI: $1.273-9.851, \quad P=0.015), \quad$ radiotherapy (OR 1.918, 95\% CI: $1.067-3.448, P=0.029)$ and acute postoperative pain within $24 \mathrm{~h}$ (OR 2.109, 95\% CI: $1.097-4.056, P=0.036$ ) were independent risk factors for the development of CPSP.

Conclusions: We found that preoperative single-shot ESPB was not associated with reduced incidence of CPSP at 1 year after BCS. ALND, radiotherapy, and acute postoperative pain within $24 \mathrm{~h}$ were independent risk factors for the development of CPSP after BCS.

Trial Registration: The study was registered in the Chinese Clinical Trial Registry (ID: ChiCTR2000038464, date of registration: September 23, 2020). 
Keywords: Erector spinae plane; Regional block; Breast cancer surgery; Chronic postsurgical pain; Neuropathic pain

\section{Key Summary Points}

Why carry out this study?

Breast cancer surgery (BCS) is associated with high incidence of chronic postsurgical pain (CPSP), which may become a heavy burden to the patient.

Erector spinae plane block (ESPB) has been applied as a new technique for analgesia in BCS patients.

We hypothesized that preoperative singleshot ESPB could reduce the incidence of CPSP at 1 year following BCS.

\section{What was learned from this study?}

This study showed that preoperative single-shot ESPB adding to general anesthesia (GA) was not associated with reduced incidence of CPSP at 1 year compared with GA alone after BCS.

Axillary lymph node dissection (ALND), radiotherapy, and acute postoperative pain within $24 \mathrm{~h}$ were independent risk factors for the development of CPSP after BCS.

Further randomized controlled studies are necessary to confirm this conclusion and more effective multimodal approaches should be considered on the prevention of CPSP after BCS.

\section{INTRODUCTION}

Being the most frequently diagnosed type of female malignancy, breast cancer accounts for a large number of cancer-related deaths worldwide [1]. Besides, patients that have undergone breast cancer surgery (BCS) are often left with chronic postsurgical pain (CPSP) with a prevalence up to $78 \%$ of the survivors [2]. CPSP may gravely impair quality of life and become a heavy burden to BCS patients $[3,4]$. The mechanism of CPSP remains unclear but several risk factors including younger age, chemotherapy and radiotherapy, axillary lymph node dissection (ALND), preoperative anxiety and depression, as well as preexisting pain conditions or poorly controlled postoperative pain have been identified in epidemiologic studies $[5,6]$.

Several regional anesthesia (RA) techniques including paravertebral block (PVB), serratus anterior plane block (SAPB), pectoral nerves (PECS) block, and the more recent erector spinae plane block (ESPB) have been applied for acute pain control after BCS [7-10]. However, the influence of RA techniques on CPSP has been sparsely investigated and remains controversial. Findings of small studies suggested that PVB might have a preventive effect on the development of CPSP [11, 12]. Unfortunately, in a recently published large randomized controlled trial, no significant impact of PVB on the incidence of CPSP and the rate of neuropathic pain (NP) had been observed [13]. ESPB is a comparatively novel interfascial plane block that targets away from the pleura, aiming to inject local anesthetics between erector spinae muscle and the thoracic transverse process [14]. It has been used as a postoperative analgesia technique in variety of surgical disciplines and shown to provide similar analgesic effects compared to PVB $[15,16]$. However, studies evaluating the effectiveness of preoperative ESPB on CPSP after BCS are still scarce.

The practice of preoperative ultrasound-guided ESPB was introduced in 2019 in our center, therefore we conducted this propensity scorematched cohort study to assess its clinical effects. We hypothesized that there would be differences in incidence of CPSP between patients who receive preoperative ultrasoundguided single-shot ESPB combined with general anesthesia (GA) and those who receive GA alone at 1 year following BCS. 


\section{METHODS}

\section{Design and Patients}

The study protocol was ethically approved by the Ethical Review Committee of Peking University People's Hospital on September 1, 2020 (No. 2020PHB222-01) and prospectively registered at http://www.chictr.org.cn with the trial identifier ChiCTR2000038464 on September 23, 2020. The study was conducted at Peking University People's Hospital in accordance with the Declaration of Helsinki. All participants provided informed consent to participate in the study.

This was an ambispective cohort study. Data were retrospectively collected on all female BCS patients aged $>18$ years and operated from October 2019 through June 2020 under general anesthesia (GA) with or without preoperative ultrasound-guided single-shot ESPB. All the enrolled patients were prospectively followed up at 1 year after surgery.

The exclusion criteria were as follows: male sex, American Society of Anesthesiologists (ASA) physical status greater than III, diagnosed mental disorder or psychiatric history, unknown type of anesthesia, unsuccessful block, bilateral breast surgery, stage IV breast cancer, coexisting malignancy, history of breast cancer surgery, reconstruction surgery, history of other chronic pain conditions.

Patients were grouped into preoperative ESPB combined with GA (ESPB + GA group) or GA alone (GA group). ESPB has been introduced in our center as a new analgesic technique for BCS patients since 2019. It was not routinely used due to patient condition or the attending anesthesiologists' preference in the time period of our record. All the operations were conducted by five surgeons experienced in BCS.

\section{Perioperative Management}

All patients having BCS routinely receive GA using a combination of intravenous (IV) propofol or etomidate, muscle relaxant and opioid based analgesia. Either a laryngeal mask airway (LMA) or endotracheal tube was used to maintain the airway. Propofol and remifentanil infusion were titrated to achieve bispectral index (BIS) value between 45 and 60, non-invasive blood pressure and heart rate within \pm $20 \%$ variation from baseline levels. Choice of volatile anesthetic agent (sevoflurane) was at the discretion of the attending anesthesiologists. Unless contraindicated, all patients received IV flurbiprofen axetil $100 \mathrm{mg}$ as a routine analgesic with tropisetron $5 \mathrm{mg}$ for postoperative nausea and vomiting (PONV) prophylaxis. Patients were transferred to the recovery room whereby postoperative pain severity was rated by anesthesia nurses using numeric rating scale (NRS) score ranging from 0 to 10 . If the patient requested analgesia or reported a NRS score of 4 or greater, IV fentanyl 50-100 $\mu \mathrm{g}$ was injected as a rescue analgesic.

\section{Ultrasound-Guided ESPB}

All blocks were performed preoperatively in the block room as an analgesic adjuvant to GA by four attending anesthesiologists with experience in ESPB. Patients were given midazolam 1-2 mg IV for sedation before blocks were performed. With the patients placed in the lateral position, a low-frequency linear ultrasound scanner (Flex Focus 500, BK Medical APS) was initiated on the spinous process in the parasagittal plane and then slid 2-3 cm laterally to make the tips of transverse process clearly visualized at T3 and T5 level. The following muscles could be seen from superficial to deep layer that were trapezius, rhomboid major, and erector spinae muscle. Then a 22-gauge block needle (100 mm, Plexu-fix; B. Braun, Germany) was inserted using in-plane technique to reach the interfascial plane between the transverse process and the erector spinae muscle. Following confirmation of the accurate position of the needle tip with 3-5 ml normal saline solution, $30 \mathrm{ml}$ of $0.4 \%$ ropivacaine was injected under ultrasound guidance. Block success was defined as reduced sensitivity to cold and pinprick stimuli as compared with the contralateral side at least one dermatomes $20 \mathrm{~min}$ after local anesthetic injection. 


\section{Data Collection and Outcome Measurements}

An independent researcher reviewed the patients' individual electronic medical documents and the regional block records, and the following data were extracted retrospectively: demographic data including age, ASA classification, weight, height, body mass index (BMI), type, duration and date of surgery, axillary surgery, postoperative acute pain score within $24 \mathrm{~h}$, postoperative rescue analgesia, use of perioperative nonsteroidal anti-inflammatories (NSAIDs), PONV, adverse events, adjuvant therapies including chemotherapy, radiotherapy, targeted therapy and endocrine therapy. Surgical type was categorized as follows: simple mastectomy, modified mastectomy, and conservative breast surgery including segmental mastectomy and skin sparing mastectomy. Axillary procedures were categorized according to whether sentinel lymph node biopsy (SLNB), ALND, or no axillary surgery was used.

Two researchers who were blinded to the group assignment interviewed the patients at 1 year postoperatively according to the date of surgery via telephone from October 1, 2020. The presence of CPSP was defined as pain that develops after the surgical procedure and persisting at least 3 months after surgery, and that was unrelated to other reasons (e.g., recurrence of disease or inflammation) [17]. The patients with CPSP were asked to locate specific regions of the pain: (1) the surgical area; (2) the axilla; (3) the ipsilateral arm; (4) the ipsilateral thorax. The brief pain inventory-short form (BPI-SF) was adopted to evaluate severity and impact of pain on daily functions $[18,19]$. Pain severity was rated on four items by an 11-point scale

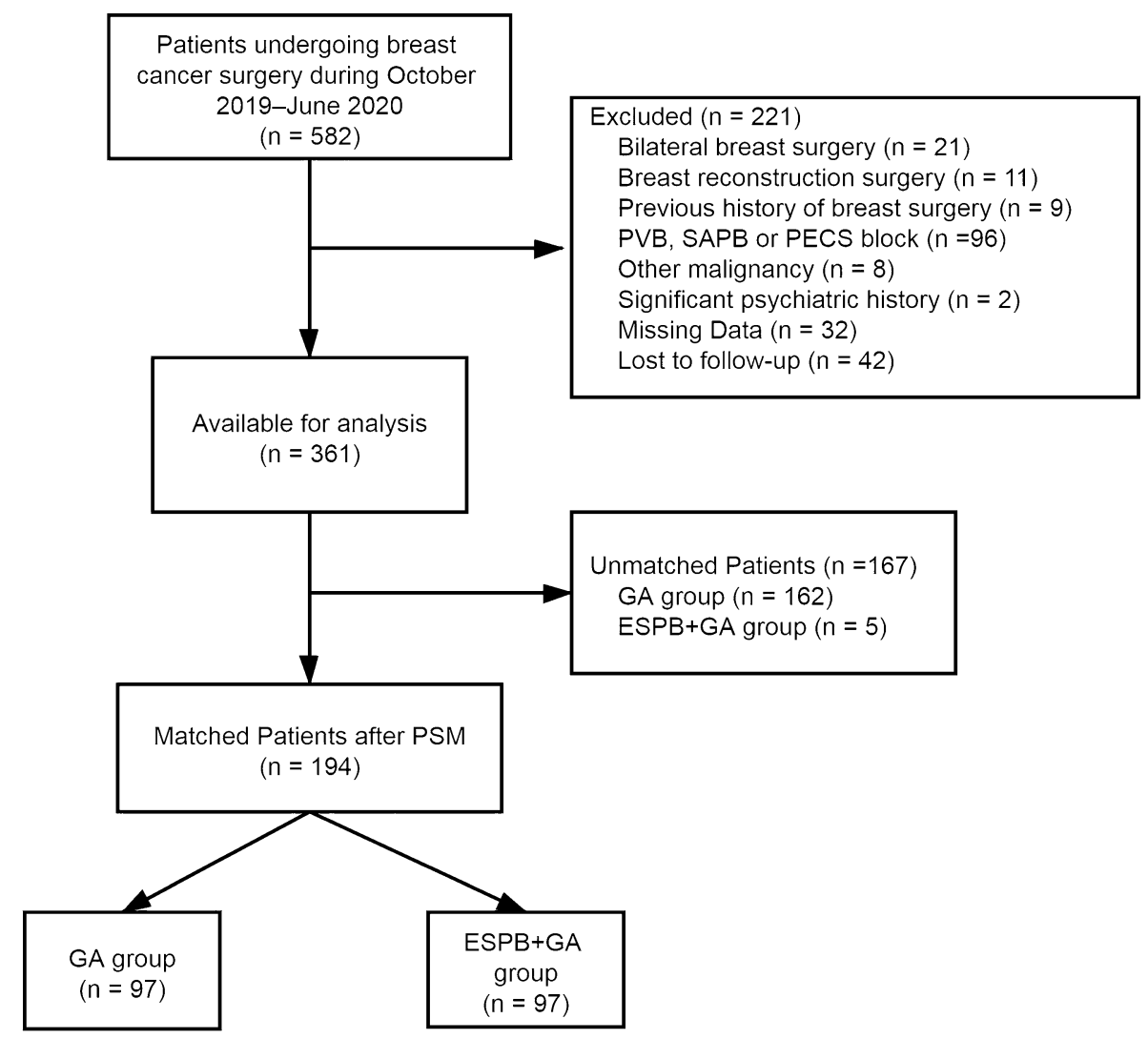

Fig. 1 Flow diagram of case selection. $P V B$ paravertebral block; $S A P B$ serratus anterior plane block; PECS pectoral nerves; $G A$ general anesthesia; $E S P B$ erector spinae plane block; PSM propensity score matching 
Table 1 Patient characteristics for the total and propensity-matched cohorts

\begin{tabular}{|c|c|c|c|c|c|c|}
\hline & \multicolumn{3}{|l|}{ Total cohort } & \multicolumn{3}{|l|}{ Matched cohort } \\
\hline & GA $(n=259)$ & $\begin{array}{l}\text { ESPB + GA } \\
(n=102)\end{array}$ & $P$ & GA $(n=97)$ & $\begin{array}{l}\text { ESPB + GA } \\
(n=97)\end{array}$ & $P$ \\
\hline Age (year) & $58.0(49.0,66.0)$ & $52.0(43.8,63.2)$ & 0.030 & $55.0(44.0,64.0)$ & $53.0(45.0,64.0)$ & 0.843 \\
\hline BMI $\left(\mathrm{kg} / \mathrm{m}^{2}\right)$ & $24.3(22.1,27.0)$ & $23.5(21.0,26.1)$ & 0.041 & $23.8(21.9,25.7)$ & $23.8(21.7,26.3)$ & 0.942 \\
\hline ASA physical status & & & 0.309 & & & 0.532 \\
\hline I & $81(31.3)$ & $39(38.2)$ & & $34(35.1)$ & $36(37.1)$ & \\
\hline II & $167(64.5)$ & $57(55.9)$ & & $60(61.9)$ & $55(56.7)$ & \\
\hline III & $11(4.2)$ & $6(5.9)$ & & $3(3.1)$ & $6(6.2)$ & \\
\hline Surgical type & & & 0.488 & & & 0.901 \\
\hline Simple mastectomy & $98(37.8)$ & $33(32.4)$ & & $33(34.0)$ & $31(32.0)$ & \\
\hline Modified radical mastectomy & $79(30.5)$ & $39(38.2)$ & & $33(34.0)$ & $36(37.1)$ & \\
\hline Breast conservation surgery & $82(31.7)$ & $30(29.4)$ & & $31(32.0)$ & $30(30.9)$ & \\
\hline Axillary surgery & & & 0.244 & & & 0.771 \\
\hline ALND & $97(37.5)$ & $45(44.1)$ & & $40(41.2)$ & $42(43.3)$ & \\
\hline No axillary surgery or SLNB & $162(62.5)$ & $57(55.9)$ & & $57(58.8)$ & $55(56.7)$ & \\
\hline Surgical time (min) & $76.0(58.0,98.0)$ & $74.5(53.0,94.2)$ & 0.272 & $76.0(58.5,97.5)$ & $74.0(53.0,94.5)$ & 0.340 \\
\hline Perioperative NSAIDs & $211(81.5)$ & $83(81.4)$ & 0.981 & $83(85.6)$ & $79(81.4)$ & 0.443 \\
\hline Chemotherapy & $153(59.1)$ & $61(59.8)$ & 0.902 & $57(58.8)$ & $56(57.7)$ & 0.877 \\
\hline Radiotherapy & $93(35.9)$ & $29(28.4)$ & 0.182 & $28(28.9)$ & $28(28.9)$ & 1.000 \\
\hline Targeted therapy & $44(17.0)$ & $16(15.7)$ & 0.764 & $16(16.5)$ & $16(16.5)$ & 1.000 \\
\hline Endocrine therapy & $168(64.9)$ & $56(54.9)$ & 0.081 & $56(57.7)$ & $55(56.7)$ & 0.880 \\
\hline
\end{tabular}

Data are presented as median (interquartile range) or number (percentage)

$G A$ general anesthesia, ESPB erector spinae plane block, BMI body mass index, $A S A$ American Society of Anesthesiologists, $A L N D$ axillary lymph node dissection, $S L N B$ sentinel lymph node biopsy, NSAIDs non-steroidal anti-inflammatory drugs

from no pain (0) to the worst pain imaginable (10). Pain interference with daily functions on seven items was also assessed on an 11-point scale $(0$, no interference and 10 complete interference). Moderate-to-severe pain was defined as an average score of 4 or greater on the single item of BPI-SF. The ID Pain scale was used as a validated assessment of NP which is a sixitem questionnaire used as a screening tool to discriminate nociceptive and neuropathic pain [20]. An ID Pain score of 2 or more was indicative of having NP. Follow-up was concluded on June 30, 2021.

The primary outcome was incidence of CPSP at 1 year following BCS. The secondary outcomes include proportion of NP, severity and interference of CPSP, acute postoperative pain within $24 \mathrm{~h}$, postoperative rescue analgesia, PONV, hospital length of stay (LOS), and adverse events (pneumothorax, hematoma, local anesthetic toxicity). 


\section{Statistical Analysis}

Data distribution was evaluated using the Shapiro-Wilk normality test. The study results for continuous variables were presented as mean \pm SD or median with interquartile range (IQR) as appropriate and analyzed with the independent samples $t$ test or Mann-Whitney $U$ test. Categorical variables were expressed as frequencies and percentages, and comparison was performed by the Chi-squared or Fisher's exact test when appropriate. Statistical analysis was conducted with SPSS 24.0 (IBM Corp., Armonk, NY, USA).

To help reduce the selection bias caused by confounding variables and the differences between the groups at baseline, propensity score matching (PSM) was performed in which we chose analgesia type as the dependent variable. The propensity score was calculated by logistic regression analysis. The following 11 confounders with a relevant effect on the study outcome due to their influence on the presence of CPSP were considered: age, BMI, ASA classification, surgical type, axillary surgery, surgical time, perioperative NSAIDs, chemotherapy, radiotherapy, targeted therapy, and endocrine therapy $[5,21]$.
The nearest neighbor method was used to match the patients in a 1:1 ratio with the caliper set at 0.1 standard difference of the estimated propensity score. The balance of baseline covariates between groups was deemed acceptable when the standardized mean difference (SMD) was below 10\%. Unpaired cases were discarded from analysis. Statistical difference was defined as a two-tailed $P<0.05$.

\section{RESULTS}

A total of 582 patients underwent BCS from October 2019 to June 2020 were investigated for eligibility, of which 361 patients (ESPB + GA group, $n=102$; GA group, $n=259$ ) were included in the analysis (Fig. 1).

The demographic characteristics and perioperative data for the total study cohort are presented in Table 1 . The sensory block upper level of ESPB was at T2 (T1-T3) and lower level at T6 (T6-T8), whereas the median number of dermatomes blocked was $3(2-4)$. Significant statistical differences were detected for age and BMI between the groups before matching. After PSM, 97 patients remained in each group. A good matching balance was achieved with all

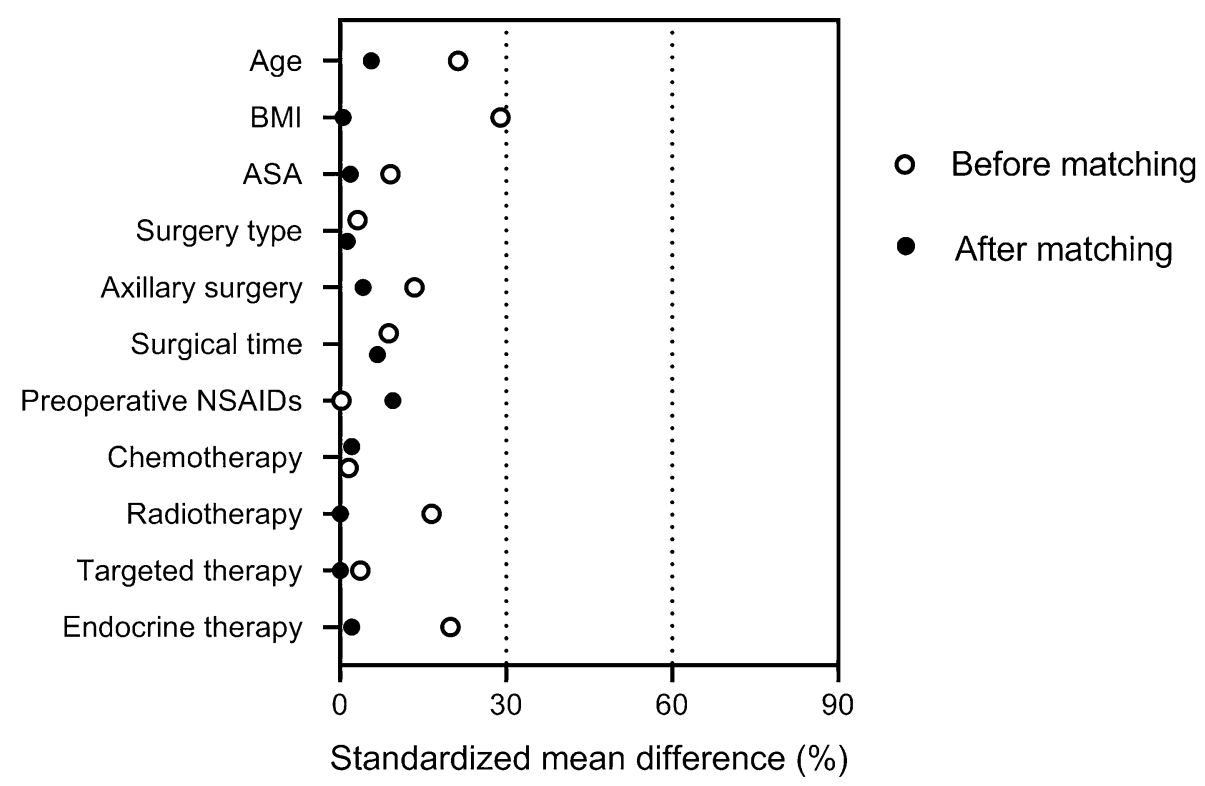

Fig. 2 Standardized mean difference of each study variable in the unmatched and matched samples. $B M I$ body mass index; ASA American Society of Anesthesiologists; NSAIDs non-steroidal anti-inflammatory drugs 
Table 2 Outcome measurements for the propensity-matched cohorts

\begin{tabular}{llll}
\hline & $\begin{array}{l}\text { GA } \\
(\boldsymbol{n}=97)\end{array}$ & $\begin{array}{l}\text { ESPB + GA } \\
(\boldsymbol{n}=\mathbf{9 7})\end{array}$ & $\mathbf{P}$ \\
\hline $\begin{array}{l}\text { Primary outcome } \\
\text { Incidence of CPSP }\end{array}$ & $32(33.0)$ & $32(33.0)$ & 1.000 \\
$\begin{array}{l}\text { Secondary outcomes } \\
\text { Proportion of NP }\end{array}$ & $14(43.8)$ & $11(34.4)$ & 0.442 \\
$\begin{array}{l}\text { Severity of CPSP } \\
\text { Interference of CPSP }\end{array}$ & $0(0,1)$ & $0(0,1)$ & 0.547 \\
$\begin{array}{l}\text { Moderate to severe } \\
\text { CPSP }\end{array}$ & $3(9.4)$ & $3(0,0)$ & 0.376 \\
$\begin{array}{l}\text { Highest acute } \\
\text { postoperative pain } \\
\text { score within 24 h }\end{array}$ & $3(2,4)$ & $2(1,2)$ & 1.000 \\
$\begin{array}{l}\text { Use of rescue } \\
\text { analgesics within }\end{array}$ & $6(6.2)$ & $2(2.1)$ & 0.043 \\
24 h & & & 0.042 \\
$\begin{array}{l}\text { PONV } \\
\text { LOS in hospital } \\
\text { (days) }\end{array}$ & $7(5,9)$ & $7(5,8)$ & 0.244 \\
\hline
\end{tabular}

Data are presented as median (interquartile range) or number (percentage)

$G A$ general anesthesia, ESPB erector spinae plane block, CPSP chronic postsurgical pain, $N P$ neuropathic pain, $P O N V$ postoperative nausea and vomiting, LOS length of stay

SMDs of the study variables were less than $10 \%$ in the matched cohort (Fig. 2).

The overall incidence of CPSP at 1 year following BCS before matching was 35.4\% (128 of 361 ), with $38.3 \%$ (49 of 128 ) satisfying the ID pain criteria for the diagnosis of NP. CPSP was reported by $74.2 \%$ (95 of 128) of the patients to be located in the surgical breast area, whereas $43.8 \%$ (56 of 128 ) in the axilla, $33.6 \%$ (43 of 128 ) in the ipsilateral arm, and $22.7 \%$ (29 of 128 ) in the ipsilateral thorax. The characteristics of pain were described as needle pain (67.5\%), burning pain $(33.2 \%)$, shooting pain $(28.5 \%)$, and numbness (9.3\%). Moderate-to-severe CPSP was reported by $11.7 \%$ (15 of 128).
After PSM, the incidence of CPSP were similar $(P=1.000)$ between the groups (Table 2$)$. There was no difference in the patients who had reported an ID pain score indicative of NP among the matched groups $(P=0.442)$. The severity of CPSP $(P=0.547)$ and interference of pain on daily functions $(P=0.376)$ did not differ between the study groups, as measured by subscale total scores on BPI-SF, respectively. There was no statistical difference $(P=1.000)$ in the rate of moderate-to-severe CPSP between the two groups (Table 2).

The highest NRS scores of acute postoperative pain within $24 \mathrm{~h}$ were significantly reduced in the ESPB + GA group comparing to the GA group $(P=0.043)$. As shown in Table 2 , postoperative rescue analgesia demand within $24 \mathrm{~h}$ was statically lower in the ESPB + GA group than in the GA group $(P=0.042)$. The incidence of PONV was significantly lower in the ESPB + GA group compared to the GA group $(P=0.031)$. There was no statistical difference $(P=0.244)$ in the hospital LOS between the two groups (Table 2). No clinical evidence of pneumothorax, hematoma, or other serious complications associated with ESPB were observed.

Considering that data of 167 patients were missing during the matching, and the incidence of CPSP was similar between the matched groups, we performed an additional logistic regression analysis on overall patients. The potential risk factors for the development of CPSP were compared in Table 3. Three significant risk factors were identified by multivariate logistic regression: ALND (OR 3.541, 95\% CI: 1.273-9.851, $P=0.015$ ), radiotherapy (OR 1.918, 95\% CI: $1.067-3.448, P=0.029)$ and acute postoperative pain within $24 \mathrm{~h}$ (OR 2.109, 95\% CI: $1.097-4.056, P=0.036)$, as shown in Table 4.

\section{DISCUSSION}

In this study, we found no significant association between preoperative single-shot ESPB and a lower incidence of CPSP at 1 year after BCS. Proportion of NP, as well as severity and interference of CPSP, were also similar for patients 
Table 3 Demographic characteristics and clinical data of patients with or without CPSP in the total cohort

\begin{tabular}{|c|c|c|c|}
\hline & $\begin{array}{l}\text { Patients with CPSP } \\
(n=128)\end{array}$ & $\begin{array}{l}\text { Patients without CPSP } \\
(n=233)\end{array}$ & $P$ \\
\hline Age (years) & & & 0.029 \\
\hline$<45$ & $18(14.1)$ & $56(24.0)$ & \\
\hline $45 \leq$ age $<65$ & $75(58.6)$ & $106(45.5)$ & \\
\hline$\geq 65$ & $35(27.3)$ & $71(30.5)$ & \\
\hline BMI $\left(\mathrm{kg} / \mathrm{m}^{2}\right)$ & & & 0.433 \\
\hline$<24.0$ & $56(43.8)$ & $115(49.4)$ & \\
\hline $24.0 \leq \mathrm{BMI}<28.0$ & $48(37.5)$ & $85(36.5)$ & \\
\hline$\geq 28.0$ & $24(18.8)$ & $33(14.2)$ & \\
\hline ASA physical status & & & 0.313 \\
\hline I & $49(38.3)$ & $71(30.5)$ & \\
\hline II & $73(57.0)$ & $151(64.8)$ & \\
\hline III & $6(4.7)$ & $11(4.7)$ & \\
\hline Surgical type & & & 0.711 \\
\hline Simple mastectomy & $46(35.9)$ & $85(36.5)$ & \\
\hline Modified radical mastectomy & $39(30.5)$ & $79(33.9)$ & \\
\hline Breast conservation surgery & $43(33.6)$ & $69(29.6)$ & \\
\hline Axillary surgery & & & 0.295 \\
\hline ALND & $55(43.0)$ & $87(37.3)$ & \\
\hline No axillary surgery or SLNB & $73(57.0)$ & $146(62.7)$ & \\
\hline Surgical time (min) & $77.5(60.2,98.8)$ & $75.0(53.5,96.5)$ & 0.158 \\
\hline Perioperative NSAIDs & $113(88.3)$ & $181(77.7)$ & 0.103 \\
\hline Chemotherapy & $83(64.8)$ & $131(56.2)$ & 0.111 \\
\hline Radiotherapy & $56(43.8)$ & $66(28.3)$ & 0.003 \\
\hline Targeted therapy & $25(19.5)$ & $35(15.0)$ & 0.271 \\
\hline Endocrine therapy & $86(67.2)$ & $138(59.2)$ & 0.136 \\
\hline Type of anesthesia & & & 0.439 \\
\hline GA & $95(74.2)$ & $164(70.4)$ & \\
\hline $\mathrm{ESPB}+\mathrm{GA}$ & $33(25.8)$ & $69(29.6)$ & \\
\hline
\end{tabular}


Table 3 continued

\begin{tabular}{lccc}
\hline & $\begin{array}{l}\text { Patients with CPSP } \\
(\boldsymbol{n}=\mathbf{1 2 8})\end{array}$ & $\begin{array}{l}\text { Patients without CPSP } \\
(\boldsymbol{n}=\mathbf{2 3 3})\end{array}$ & $\boldsymbol{P}$ \\
\hline $\begin{array}{l}\text { Highest acute postoperative pain score within } \\
24 \mathrm{~h}\end{array}$ & $3(2,4)$ & $2(2,3)$ & 0.028 \\
\hline
\end{tabular}

Data are presented as median (interquartile range) or number (percentage)

CPSP chronic postsurgical pain, BMI body mass index, ASA American Society of Anesthesiologists, ALND axillary lymph node dissection, $S L N B$ sentinel lymph node biopsy, NSAIDs non-steroidal anti-inflammatory drugs, $G A$ general anesthesia, $E S P B$ erector spinae plane block

receiving ESPB combined with GA and GA alone.

The incidence of CPSP after BCS varied from 25 to $78 \%$ in previous studies [2]. This may partially be attributed to different definitions of chronic pain. Our study showed that the overall incidence of CPSP was $35.4 \%$ at 1 year after BCS, which was within the range of previously reported incidences. Although the mechanism of CPSP remains unclear, evidence has suggested that peripheral and central sensitization may play an important role in the development of chronic pain after surgery [17]. Theoretically, RA could alleviate acute postoperative pain by blocking peripheral nociceptive input into the spinal cord, which contributed to spinal sensitization. Previous studies have reported that PVB and PECS could reduce incidence of chronic pain after breast surgery [22-24]. In a recent meta-analysis, the use of single-shot PVB has been shown to be protective against CPSP at 6 months following BCS, but the studies included are weakened by methodological limitations [12]. However, our study found that the effect of a single-shot ESPB might be limited on prevention of CPSP at 1 year after BCS. The contrasting results may be partially explained by different RA techniques used in our study and the previous ones. In fact, differences in analgesic effects between the block techniques for BCS patients have been found in some studies [25-27], which indicates that ESPB and PVB may have different mechanisms of action $[28,29]$. The most possible mechanism of analgesia for ESPB is a direct effect of local anesthetics via diffusion to the erector spinae muscle within the costotransverse foramen region with possible spread to the paravertebral space which is the main target of PVB [30]. However, the extent of craniocaudal spread to the adjacent tissue compartments and the sensory blockade of ESPB varied greatly. Our study showed a median number of anesthetized dermatomes of 3 . which was comparatively smaller than other studies that reported significant local anesthetic distribution with a single ESPB injection [14].

The high risk of NP after BCS might be another possible explanation for our findings on the primary outcome. In agreement to previous studies, we found that $38.3 \%$ of the patients who developed chronic pain after BCS described a neuropathic component [31, 32]. Moreover, our study also confirmed that a substantial proportion of patients reported CPSP as moderate to severe in nature, which is in accordance with previous study [2]. Due to the cancer-related multimodal treatment, chronic pain after BCS is often described as a mixed pain, which consists predominantly of a neuropathic nature and partially related to intraoperative nerve injury [33, 34]. Although RA may prevent chronic pain after certain types of surgeries with a low risk of NP, the situation may be different for surgeries with a high risk of NP, such as BCS [23].

Although no association of ESPB and reduced incidence of CPSP was found in our study, we still confirmed that ESPB markedly improve postoperative analgesia and reduce rescue analgesics after BCS, which is in line with the existing literature $[25,35]$. In addition, the 
Table 4 Multivariate logistic regression analysis for the development of CPSP in the total cohort

\begin{tabular}{|c|c|c|c|}
\hline & OR & $95 \% \mathrm{CI}$ & $P$ \\
\hline \multicolumn{4}{|l|}{ Age (years) } \\
\hline$<45$ & 2.136 & $0.923-4.940$ & 0.076 \\
\hline $45 \leq$ Age $<65$ & 0.870 & $0.478-1.581$ & 0.648 \\
\hline$\geq 65$ & Reference & & \\
\hline \multicolumn{4}{|l|}{ BMI $\left(\mathrm{kg} / \mathrm{m}^{2}\right)$} \\
\hline$<24.0$ & 1.867 & $0.922-3.780$ & 0.083 \\
\hline $24.0 \leq \mathrm{BMI}<28.0$ & 1.716 & $0.852-3.458$ & 0.131 \\
\hline$\geq 28.0$ & Reference & & \\
\hline \multicolumn{4}{|l|}{ ASA physical status } \\
\hline I & 0.480 & $0.145-1.591$ & 0.230 \\
\hline II & 0.901 & $0.298-2.723$ & 0.853 \\
\hline III & Reference & & \\
\hline \multicolumn{4}{|l|}{ Surgical type } \\
\hline Simple mastectomy & 0.706 & $0.360-1.383$ & 0.311 \\
\hline Modified radical mastectomy & 1.909 & $0.719-4.766$ & 0.108 \\
\hline Breast conservation surgery & Reference & & \\
\hline \multicolumn{4}{|l|}{ Axillary surgery } \\
\hline ALND & 3.541 & $1.273-9.851$ & 0.015 \\
\hline No axillary surgery or SLNB & Reference & & \\
\hline Surgical time (min) & 0.995 & $0.986-1.004$ & 0.295 \\
\hline Perioperative NSAIDs & 1.109 & $0.970-3.056$ & 0.125 \\
\hline Chemotherapy & 1.041 & $0.573-1.890$ & 0.895 \\
\hline Radiotherapy & 1.918 & $1.067-3.448$ & 0.029 \\
\hline Targeted therapy & 1.266 & $0.659-2.432$ & 0.479 \\
\hline Endocrine therapy & 1.325 & $0.800-2.195$ & 0.274 \\
\hline \multicolumn{4}{|l|}{ Type of anesthesia } \\
\hline GA & 1.042 & $0.614-1.768$ & 0.880 \\
\hline $\mathrm{ESPB}+\mathrm{GA}$ & Reference & & \\
\hline Acute postoperative pain score within $24 \mathrm{~h}$ & 2.109 & $1.097-4.056$ & 0.036 \\
\hline
\end{tabular}

$O R$ odds ratio, $C I$ confidence interval, $B M I$ body mass index, $A S A$ American Society of Anesthesiologists, SLNB sentinel lymph node biopsy, $A L N D$ axillary lymph node dissection, NSAIDs non-steroidal anti-inflammatory drugs, $G A$ general anesthesia, ESPB erector spinae plane block 
rate of PONV and postoperative rescue analgesia were significantly differed between the two study groups, which may partly demonstrate the opioid-sparing effect of ESPB and its potential to facilitate recovery. PVB has been demonstrated to be one of the most effective RA techniques for acute postoperative pain management. However, it is also challenging to perform the block proximate to the pleura and central neuraxial system. ESPB is a relatively safer method which target away from the pleura. In our study, no episodes of pneumothorax, epidural administration and other serious complications that related to nerve block were observed, suggesting the safety of ESPB under ultrasound guidance with reduced risks associated with conventional thoracic epidural analgesia (TEA) and PVB.

The etiology of CPSP is multifactorial. Consistent with previous studies exploring predictors of chronic pain after BCS, our study suggests that ALND, radiotherapy, and acute postoperative pain appear to be the risk factors of CPSP $[6,36]$. Thus, multimodal approaches including both surgical and anesthetic techniques should be utilized for the purpose of minimizing the occurrence of CPSP. The effects of perioperative pregabalin, ketamine therapy, and lidocaine infusion on prevention of CPSP after BCS have also been investigated in some randomized trials, which showed promising results [37-39]. Therefore, further studies based on multimodal strategies will be needed, considering the high rates of CPSP and the high proportion of NP after BCS.

There are several limitations to our study. First, due to the non-randomized design and monocentric nature of this study, the results only indicate association between the covariates of interest and may not be representative in larger population. Second, although PSM method could help lower the risk of confounding bias and selection bias, we could only account for confounders of which relevant data were documented. For instance, psychological status as well as pain threshold should be taken into consideration. Third, different techniques of ESPB may change the effects of the block. That said, the number of injection levels, type, volume, and concentration of local anesthetics might affect the sensory blockade and analgesic effects. Fourth, we did not compare the effects of ESPB with other blocks on CPSP after BCS. Fifth, due to the complex sensory innervation of the breast and nipple area, nerves other than upper thoracic spinal nerves may be involved, making a single block with ESPB less effective. Conversely, the main strength of this study was being the first study to evaluate effects of ESPB on chronic pain at 1 year in patients undergoing BCS.

\section{CONCLUSIONS}

In conclusion, our results revealed that a preoperative single shot of ESPB combined with GA was not associated with lower incidence of CPSP at 1 year compared with GA alone after BCS. ALND, radiotherapy, and acute postoperative pain within $24 \mathrm{~h}$ were independent risk factors for the development of CPSP after BCS. However, the limitations of the study do not permit us to be conclusive on this argument. Further well-conducted randomized controlled studies should be needed to confirm the results.

\section{ACKNOWLEDGEMENTS}

We are grateful to the faculties of the Department of Anesthesiology for their constant support and guidance to conduct this study. We also thank all the participants for their involvement in the study.

Funding. No funding or sponsorship was received for this study or publication of this article. The Rapid Service Fee was funded by the authors.

Authorship. All named authors meet the International Committee of Medical Journal Editors (ICMJE) criteria for authorship for this article, take responsibility for the integrity of the work as a whole, and have given their approval for this version to be published.

Authors' Contributions. Conceptualization and methodology: Ling Xin, Yi Feng; Formal 
analysis and investigation: Ling Xin, Ning Hou, Ziyan Zhang; Writing-original draft preparation: Ling Xin, Ning Hou, Ziyan Zhang; Writing-review and editing: Yi Feng; Resources: Ning Hou; Supervision: Yi Feng.

Disclosures. Ling Xin, Ning Hou, Ziyan Zhang, and Yi Feng declare that they have no conflict of interest.

Compliance with Ethics Guidelines. The study was conducted at Peking University People's Hospital in accordance with the Declaration of Helsinki. The study protocol was approved by the Ethical Review Committee of Peking University People's Hospital (No. 2020PHB222-01). All participants provided informed consent to participate in the study.

Data Availability. The datasets generated during and/or analyzed during the current study are available from the corresponding author upon reasonable request.

Open Access. This article is licensed under a Creative Commons Attribution-NonCommercial 4.0 International License, which permits any non-commercial use, sharing, adaptation, distribution and reproduction in any medium or format, as long as you give appropriate credit to the original author(s) and the source, provide a link to the Creative Commons licence, and indicate if changes were made. The images or other third party material in this article are included in the article's Creative Commons licence, unless indicated otherwise in a credit line to the material. If material is not included in the article's Creative Commons licence and your intended use is not permitted by statutory regulation or exceeds the permitted use, you will need to obtain permission directly from the copyright holder. To view a copy of this licence, visit http://creativecommons.org/licenses/by$\mathrm{nc} / 4.0 /$.

\section{REFERENCES}

1. Siegel RL, Miller KD, Jemal A. Cancer statistics, 2020. CA Cancer J Clin. 2020;70:7-30.

2. Wang L, Cohen JC, Devasenapathy N, et al. Prevalence and intensity of persistent post-surgical pain following breast cancer surgery: a systematic review and meta-analysis of observational studies. $\mathrm{Br} \mathrm{J}$ Anaesth. 2020;125:346-57.

3. Saporito A, Aguirre J, Borgeat A, et al. Persistent post-discharge pain and chronic postoperative pain after breast cancer surgery under general anesthesia and single-shot paravertebral block: incidence, characteristics and impact on quality of life and healthcare costs. J Pain Res. 2019;12:1193-9.

4. Hamood R, Hamood H, Merhasin I, et al. Chronic pain and other symptoms among breast cancer survivors: prevalence, predictors, and effects on quality of life. Breast Cancer Res Treat. 2018;167: 157-69.

5. Wang L, Guyatt GH, Kennedy SA, et al. Predictors of persistent pain after breast cancer surgery: a systematic review and meta-analysis of observational studies. CMAJ. 2016;188:E352-61.

6. Andersen KG, Duriaud HM, Jensen HE, et al. Predictive factors for the development of persistent pain after breast cancer surgery. Pain. 2015;156: 2413-22.

7. Hetta DF, Rezk KM. Pectoralis-serratus interfascial plane block vs thoracic paravertebral block for unilateral radical mastectomy with axillary evacuation. J Clin Anesth. 2016;34:91-7.

8. Singh S, Chowdhary NK. Erector spinae plane block an effective block for postoperative analgesia in modified radical mastectomy. Indian J Anaesth. 2018;62:148-50.

9. Mazzinari G, Rovira L, Casasempere A, et al. Interfascial block at the serratus muscle plane versus conventional analgesia in breast surgery: a randomized controlled trial. Reg Anesth Pain Med. 2019;44:52-8.

10. Neethu M, Pandey RK, Sharma A, et al. Pectoral nerve blocks to improve analgesia after breast cancer surgery: a prospective, randomized and controlled trial. J Clin Anesth. 2018;45:12-7.

11. Heesen M, Klimek M, Rossaint R, et al. Paravertebral block and chronic postsurgical pain after breast surgery: meta-analysis and trial sequential analysis. Anaesthesia. 2016;71:1471-81. 
12. Hussain N, Shastri U, McCartney CJL, et al. Should thoracic paravertebral blocks be used to prevent chronic postsurgical pain after breast cancer surgery? A systematic analysis of evidence in light of IMMPACT recommendations. Pain. 2018;159: 1955-7.

13. Sessler DI, Pei L, Huang Y, et al. Recurrence of breast cancer after regional or general anaesthesia: a randomised controlled trial. Lancet. 2019;394: 1807-15.

14. Forero M, Adhikary SD, Lopez $\mathrm{H}$, et al. The erector spinae plane block: a novel analgesic technique in thoracic neuropathic pain. Reg Anesth Pain Med. 2016;41:621-7.

15. Saadawi M, Layera S, Aliste J, et al. Erector spinae plane block: a narrative review with systematic analysis of the evidence pertaining to clinical indications and alternative truncal blocks. J Clin Anesth. 2021;68:110063.

16. Leong RW, Tan ESJ, Wong SN, et al. Efficacy of erector spinae plane block for analgesia in breast surgery: a systematic review and meta-analysis. Anaesthesia. 2021;76:404-13.

17. Treede RD, Rief W, Barke A, et al. Chronic pain as a symptom or a disease: the IASP classification of chronic pain for the International Classification of Diseases (ICD-11). Pain. 2019;160:19-27.

18. Im DD, Jambaulikar GD, Kikut A, et al. Brief Pain Inventory-Short Form: a new method for assessing pain in the emergency department. Pain Med. 2020;21:3263-9.

19. de Andrés AJ, Cruces Prado LM, Canos Verdecho MA, et al. Validation of the Short Form of the Brief Pain Inventory (BPI-SF) in Spanish patients with non-cancer-related pain. Pain Pract. 2015;15: 643-53.

20. Reyes-Gibby C, Morrow PK, Bennett MI, et al. Neuropathic pain in breast cancer survivors: using the ID Pain as a screening tool. J Pain Symptom Manag. 2010;39:882-9.

21. Spivey TL, Gutowski ED, Nantthasorn Z, et al. Chronic pain after breast surgery: a prospective, observational study. Ann Surg Oncol. 2018;25:1-8.

22. Lin ZM, Li MH, Zhang F, et al. Thoracic paravertebral blockade reduces chronic postsurgical pain in breast cancer patients: a randomized controlled trial. Pain Med. 2020;21:3539-47.

23. Qian B, Fu S, Yao Y, et al. Preoperative ultrasoundguided multilevel paravertebral blocks reduce the incidence of postmastectomy chronic pain: a double-blind, placebo-controlled randomized trial. J Pain Res. 2019;12:597-603.

24. De Cassai A, Bonanno C, Sandei L, et al. PECS II block is associated with lower incidence of chronic pain after breast surgery. Korean J Pain. 2019;32: 286-91.

25. Gürkan Y, Aksu C, Kuş A, et al. Erector spinae plane block and thoracic paravertebral block for breast surgery compared to IV-morphine: a randomized controlled trial. J Clin Anesth. 2020;59:84-8.

26. Swisher MW, Wallace AM, Sztain JF, et al. Erector spinae plane versus paravertebral nerve blocks for postoperative analgesia after breast surgery: a randomized clinical trial. Reg Anesth Pain Med. 2020;45:260-6.

27. Altıparmak B, Korkmaz TM, Uysal Aİ, et al. Comparison of the effects of modified pectoral nerve block and erector spinae plane block on postoperative opioid consumption and pain scores of patients after radical mastectomy surgery: a prospective, randomized, controlled trial. J Clin Anesth. 2019;54:61-5.

28. Ivanusic J, Konishi Y, Barrington MJ. A cadaveric study investigating the mechanism of action of erector spinae blockade. Reg Anesth Pain Med. 2018;43:567-71.

29. Schoenfeldt J, Guffey R, Fingerman M. Cadaveric study investigating the mechanism of action of erector spinae blockade. Reg Anesth Pain Med. 2019;44:280.

30. Schwartzmann A, Peng P, Maciel MA, et al. A magnetic resonance imaging study of local anesthetic spread in patients receiving an erector spinae plane block. Can J Anaesth. 2020;67:942-8.

31. Fontes F, Pereira S, Castro-Lopes JM, et al. A prospective study on the neurological complications of breast cancer and its treatment: updated analysis three years after cancer diagnosis. Breast. 2016;29:31-8.

32. Fuzier R, Puel F, Izard P, et al. Prospective cohort study assessing chronic pain in patients following minor surgery for breast cancer. J Anesth. 2017;31: 246-54.

33. Nijs J, Leysen L, Adriaenssens N, et al. Pain following cancer treatment: guidelines for the clinical classification of predominant neuropathic, nociceptive and central sensitization pain. Acta Oncol. 2016;55:659-63.

34. Wijayasinghe $\mathrm{N}$, Duriaud HM, Kehlet $\mathrm{H}$, et al. Ultrasound guided intercostobrachial nerve blockade in patients with persistent pain after breast 
cancer surgery: a pilot study. Pain Physician. 2016;19:E309-18.

35. Yao Y, Li H, He Q, et al. Efficacy of ultrasoundguided erector spinae plane block on postoperative quality of recovery and analgesia after modified radical mastectomy: randomized controlled trial. Reg Anesth Pain Med. 2020;45:5-9.

36. Habib AS, Kertai MD, Cooter M, et al. Risk factors for severe acute pain and persistent pain after surgery for breast cancer: a prospective observational study. Reg Anesth Pain Med. 2019;44:192-9.

37. Khan JS, Hodgson N, Choi S, et al. Perioperative pregabalin and intraoperative lidocaine infusion to reduce persistent neuropathic pain after breast cancer surgery: a multicenter, factorial, randomized, controlled pilot trial. J Pain. 2019;20:980-93.

38. Kang C, Cho AR, Kim KH, et al. Effects of intraoperative low-dose ketamine on persistent postsurgical pain after breast cancer surgery: a prospective, randomized, controlled, double-blind study. Pain Physician. 2020;23:37-47.

39. Reyad RM, Omran AF, Abas DN, et al. The possible preventive role of pregabalin in postmastectomy pain syndrome: a double-blinded randomized controlled trial. J Pain Symptom Manage. 2019;57:1-9. 